\title{
Effect of roughness on pressure fluctuations in a turbulent channel flow
}

\author{
Kiran Bhaganagar ${ }^{\mathrm{a})}$ \\ Department of Mechanical Engineering, University of Maine, Orono, Maine 04469 \\ Gary Coleman \\ School of Engineering Sciences/Aeronautics \& Astronautics, University of Southampton, \\ Southampton, United Kingdom \\ John Kim \\ Department of Mechanical and Aerospace Engineering, University of California, Los Angeles, \\ Los Angeles, California 90095
}

(Received 25 October 2006; accepted 22 December 2006; published online 16 February 2007)

\begin{abstract}
Direct numerical simulation is used to investigate the nature of pressure fluctuations induced by surface roughness in a turbulent channel flow at $\operatorname{Re}_{\tau}=400$ for three-dimensional periodic roughness elements, whose peaks overlap approximately $25 \%$ of the logarithmic layer. The three-dimensional roughness elements alter the pressure statistics significantly, compared to the corresponding smooth-wall flow, in both the inner and outer (core) regions of the channel. The direct consequence of roughness is an increased form drag, associated with more intense pressure fluctuations. However, it also alters the pressure fluctuations in the outer layer of the flow, and modifies the length scales defined by two-point correlations. We also find that the depth of the roughness sublayer defined by the pressure fluctuations is very different from that given by the large- and small-scale statistics from the velocity field. (C) 2007 American Institute of Physics. [DOI: 10.1063/1.2482883]
\end{abstract}

This paper focuses on the influence of surface roughness on pressure fluctuations for a better understanding of inner/ outer layer interactions in wall-bounded turbulence. We consider incompressible turbulent flow in a plane channel, the lower wall of which is covered by a regular array of threedimensional "egg-carton"-shaped elements. ${ }^{1}$ The elements extend well above the viscous sublayer of the equivalent smooth-wall flow, with their peak-to-valley height $h$ given by $h^{+}=h u_{\tau}^{s} / \nu=21.6$ (based on the friction velocity from the smooth-wall side of the channel, $u_{\tau}^{s}$ ), corresponding to $h$ $=0.054 \delta$ ( $\delta$ is the half-height of the channel). The roughness is prescribed via a virtual no-slip surface whose mean height is at $y=-0.96 \delta$, using an immersed boundary technique. For the Reynolds number used here, $\operatorname{Re}_{\tau}=400$, the halfwidth-toroughness ratio is $\delta / h \approx 18.5$. Assuming the top of the logarithmic layer reaches to about $0.2 \delta$, this implies that the peaks of the roughness elements extend approximately onequarter of the way into the logarithmic region. As a consequence, the roughness can be expected to directly affect the bottom of the logarithmic layer but not completely destroy it. This case is relevant in a number of engineering and especially meteorological contexts.

Much remains to be learned about rough-wall boundary layers. In light of existing experimental results, it now appears that rough-wall boundary layers can be categorized according to whether the surface roughness does not affect the outer layer ${ }^{2,3}$ or if it does affect the outer layer. ${ }^{1,4}$ One of the most important tasks is to distinguish between effects that are local to the individual roughness elements and those that are manifest throughout the flow. Another is to determine reliable measures of these effects, and thereby ascertain the

${ }^{\text {a)} E l e c t r o n i c ~ m a i l: ~ k i r a n . b h a g a n a g a r @ m a i n e . e d u ~}$ essential metrics, the flow parameters, needed to completely define the behavior of rough-wall flows. In this study, these tasks are addressed by investigating pressure fluctuations in a plane channel with one smooth and one rough wall. Because of the global nature of the pressure field in any incompressible flow, this approach is expected to be a particularly effective way of quantifying roughness-induced features that are projected into the outer layer (above the roughness sublayer).

Given the difficulties associated with measuring pressure within a turbulent flow, it is not surprising that the current picture of rough-wall boundary layers has been derived primarily from velocity and vorticity statistics (see, e.g., Refs. $1-3)$. On the other hand, there have been some useful experimental and numerical studies based on pressure fluctuations at the surface. Most have focused on the enhanced form drag induced by pressure forces acting upon roughness elements of various types. ${ }^{5-7}$ A somewhat different approach was taken by Mulhearn, ${ }^{8}$ who examined the influence of a step increase in roughness height (from slightly to fully rough conditions, for two-dimensional transverse rectangular-grove $k$-type elements) upon the surface pressure intensity and spectra. By comparing these results with those from the smooth-wall case, it was inferred that the interaction between the inner and outer layers is qualitatively different for the rough-wall flow, in that only for the rough-wall case is the surface-pressure signature affected by the details of the outer region. This underlines the value of using pressure statistics as a metric to classify the critical flow and surface-geometry parameters for rough-wall boundary layers.

A direct numerical simulation (DNS) tool has been developed to simulate turbulent flow over a complex boundary using an immersed boundary method, ${ }^{1}$ thus retaining the 

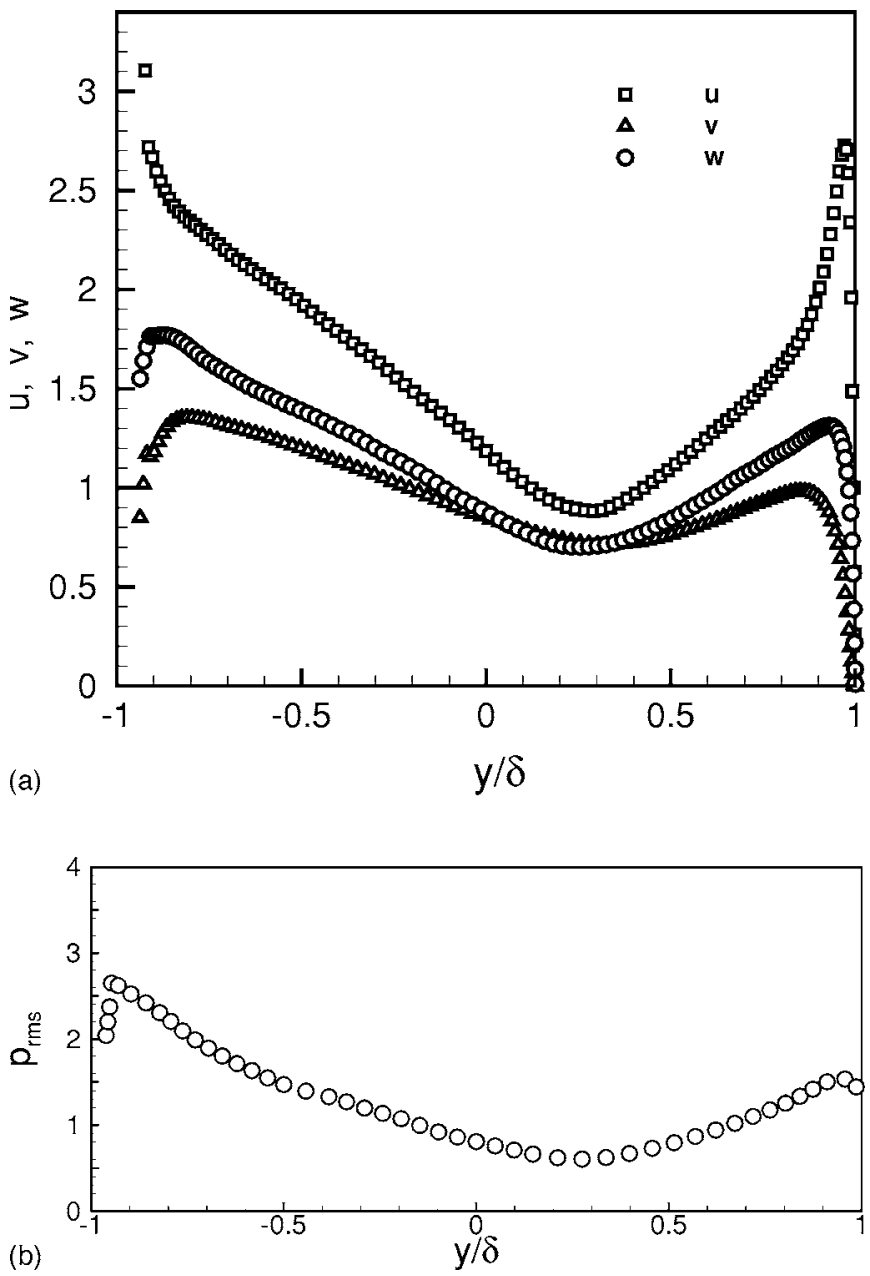

FIG. 1. Root-mean-square fluctuations of (a) velocity and (b) pressure normalized by the smooth-wall friction velocity $u_{\tau}^{s}$.

simplicity and efficiency of computation in a Cartesian system. The Navier-Stokes equations were solved in the vertical-velocity and vertical-vorticity formulation. Further details of the implementation are described in Ref. 1. The simulations have been performed in a periodic channel of streamwise and spanwise size $L_{x} / \delta=2 \pi$ and $L_{z} / \delta=\pi$. The spatial discretization used 256 streamwise Fourier modes, 257 wall-normal compact finite-difference grid points of fourth-order accuracy, and 256 spanwise Fourier modes. Pressure fields have been computed as post-processing by solving the pressure Poisson equation.

Figures 1(a) and 1(b) show root-mean-square (rms) velocity and pressure fluctuations across the channel, nondimensionalized by the friction velocity from the smooth wall. The magnitude of the pressure fluctuations at the smooth wall agrees well with the earlier (dual-smooth-wall) channel DNS of Kim et al. ${ }^{9,10}$ Fluctuation levels of all three velocity components and especially the pressure are larger adjacent to the rough wall than they are at corresponding distances from the smooth one. This illustrates the increased turbulence intensity induced by the roughness, in agreement with many previous experimental and numerical studies. We define an effective layer thickness $\delta_{t}$ for each side based on the distance from the virtual (or actual, for the smooth wall) origin
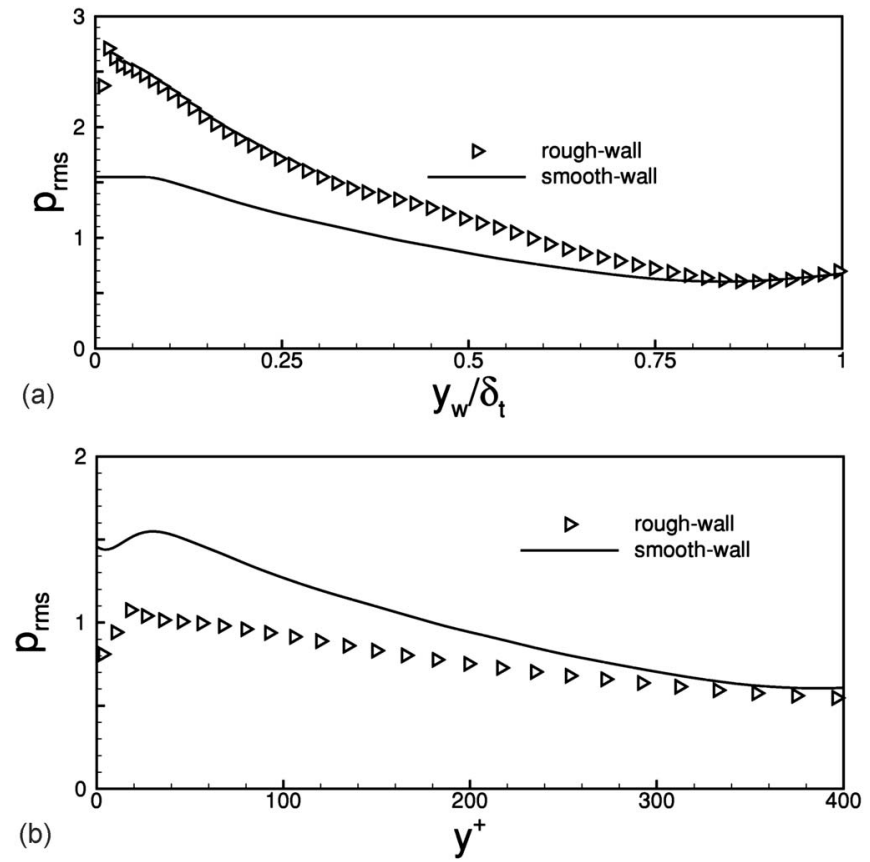

FIG. 2. Rms pressure fluctuations (a) $p$ scaled by smooth-wall friction velocity $\left(u_{\tau}^{s}\right)^{2}$ and plotted in outer-layer units and (b) $p$ scaled by rough-wall friction velocity $\left(u_{\tau}^{r}\right)^{2}$ for rough-wall and smooth-wall friction velocity $\left(u_{\tau}^{s}\right)^{2}$ for the smooth-wall and plotted in inner-layer units.

to the location of the minimum velocity fluctuations $(y / \delta$ $\approx+0.3$ ). This thickness (which is $\delta_{t} / \delta \approx 0.7$ and 1.3 , respectively, for the smooth and rough-wall sides) is used for the outer-layer scaling in Fig. 2(a). The outer-layer velocity scale is taken to be the smooth-side friction velocity $u_{\tau}^{s}$. When scaled in outer units, the pressure fluctuations are modified both in the near and outer layer of the channel. The pressure fluctuation level at the wall is enhanced by the roughness, by approximately the same factor as it increases the streamwise surface stress $\left(u_{\tau}^{r} / u_{\tau}^{s}\right.$ is 1.61). A similar trend was observed in the experiments of Blake. ${ }^{11}$ In turbulence bounded by a smooth wall, the largest amplitude wall-pressure fluctuations are thought to be linked with quasistreamwise vortices and near-wall kinetic energy production. ${ }^{12}$ Further, $\mathrm{Kim}^{9}$ has related the strength of the streamwise vortices to the source term of the pressure fluctuations in the pressure Poisson equation. This is consistent in the rough-wall scenario as the rms of $\omega_{x}$ near the rough wall is substantially larger. The fact that the ratio of the (off-wall) peak pressure fluctuations to the wall pressure on the rough-wall side is significantly higher than the corresponding ratio on the smooth wall side suggests that the roughness has a profound influence on the instantaneous structure of the near-wall turbulence. A structural change is also implied by the inner scaling [Fig. 2(b)]: although the pressure fluctuations are larger on the roughwall side in absolute terms, when scaled in wall units they represent a smaller fraction of the local $u_{\tau}$ compared to the smooth-wall side. These results demonstrate that based on the pressure statistics, both the inner and outer layer of the channel are affected by the roughness.

We now use the pressure field to examine the depth $\zeta$ of the roughness sublayer, within which the flow is directly af- 


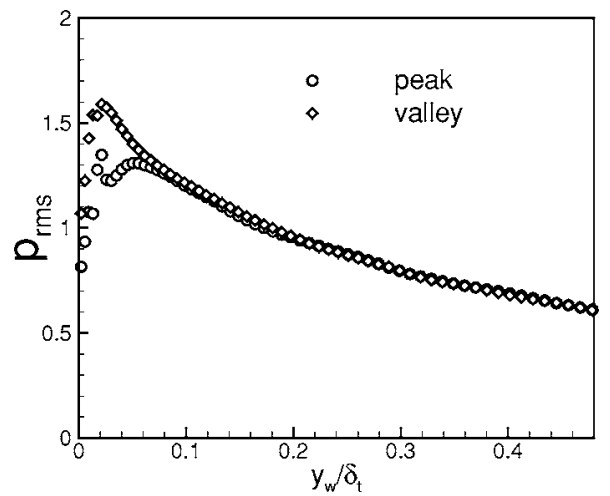

FIG. 3. Profiles of rms pressure fluctuations scaled by rough-wall friction velocity above peak and valley locations of the roughness elements.

fected by the individual roughness elements, such that timeaveraged statistics in this region are not independent of the location at the same mean wall-normal distance (i.e., they are not spatially homogeneous). In an earlier analysis of this flow, ${ }^{1}$ we discovered that $\zeta$ differs according to whether it is defined based on large- or small-scale velocity statistics. The large-scale measure, $\zeta_{q}$ (based on rms velocity fluctuations), is about $1.5 h$, while $\zeta_{\omega}$, the small-scale (vorticity-based) sublayer depth, is about $1.1 h$ (recall that $h$ is the valley-to-peak height of the roughness elements).

Determining which of $\zeta_{q}$ or $\zeta_{\omega}$ is closest to the pressurebased depth $\zeta_{p}$ should cast light on the structure and mechanisms of the turbulence adjacent to the roughness. To calculate $\zeta_{p}$, we compare the wall-normal variation of the pressure fluctuations directly above distinct features of the roughness pattern - in this case, the highest (peak) and lowest (valley) points of the double-sine roughness distribution. The rms pressure profiles for these two locations are shown in Fig. 3. Assuming that the statistics at these two locations represent the extremes, we find that spatial homogeneity is not achieved until $y / \delta=-0.84$. This corresponds to $\zeta_{p} \approx 2 h$. Al-
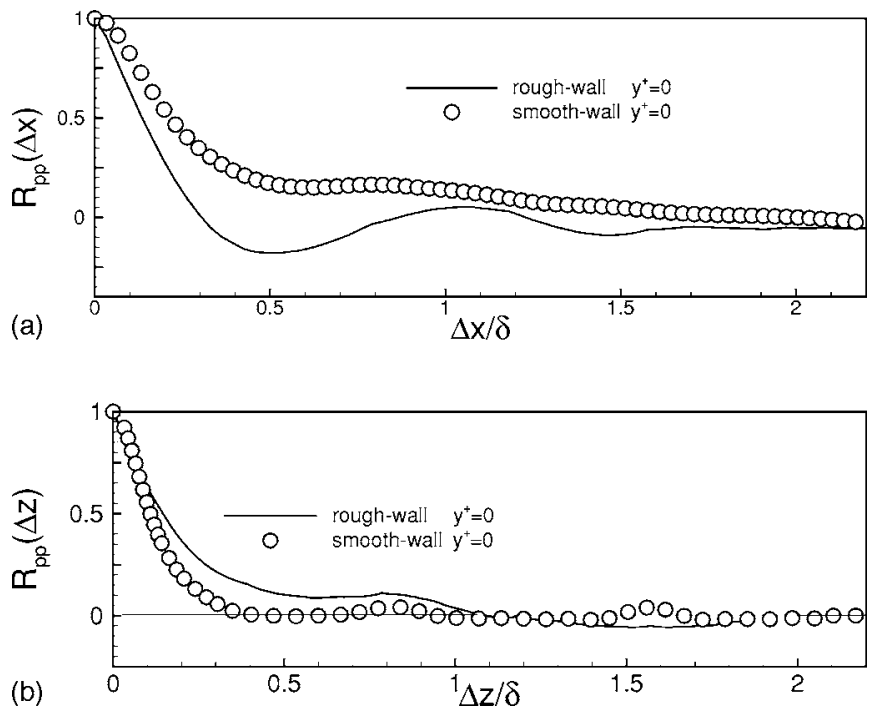

FIG. 4. Two-point pressure-pressure correlation at $y^{+}=0$. (a) Variation of $R_{p p}(\Delta x)$ in the streamwise direction and (b) variation of $R_{p p}(\Delta z)$ in the spanwise direction.

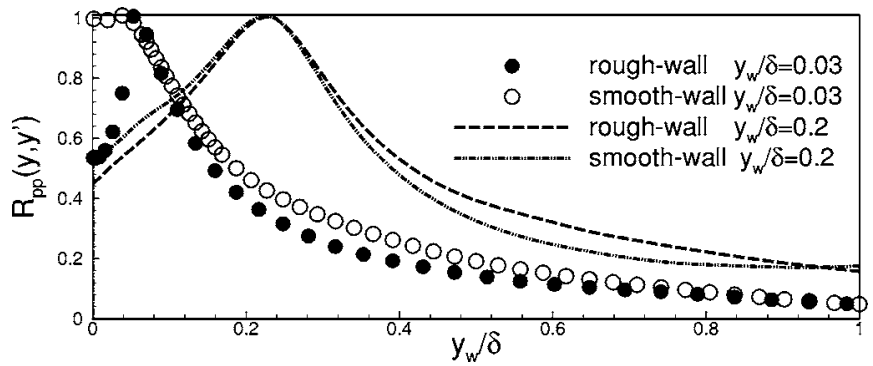

FIG. 5. Two-point correlations $R_{p p}\left(y, y^{\prime}\right)$ along the wall-normal direction at $y_{w} / \delta=0.03$ and 0.21 .

though the sublayer depth $\zeta_{p}$ defined by the pressure fluctuations is closer to that given by the large-scale rms velocity profiles $\left(\zeta_{q}\right)$ than the vorticity-based counterpart $\left(\zeta_{\omega}\right)$, it is nevertheless significantly larger than both $\zeta_{q}$ and especially $\zeta_{\omega}$-an observation with significant turbulence modeling consequence. This demonstrates that the basic global or integral characteristics for pressure-based statistics are different compared to large-scale velocity or small-scale vorticity statistics.

Further length-scale information is provided in Figs. 4(a) and 4(b), which illustrate, respectively, the two-point correlations of pressure-pressure fluctuations in the streamwise and spanwise directions at $y^{+}=0$, for both the smooth- and rough-wall sides. The streamwise correlations for the roughand smooth-wall flows [Fig. 4(a)] reveal that the streamwise extent of these structures decreases due to roughness. Compared to the smooth-wall baseline, the implied spanwise integral length scale is larger for the region above the rough surface. It is interesting that the spanwise length scales of the pressure field are affected by the roughness, even though the corresponding spanwise length scales for the velocity are not significantly altered. ${ }^{1}$ This adds to the body of evidence that the structures of the velocity and pressure fluctuations are qualitatively different. As can be seen from wall-normal correlations [defined as $R_{p p}\left(y, y^{\prime}\right)$ $\left.=\left\langle p(x, y, z, t) p\left(x, y^{\prime}, z, t\right)\right\rangle / p_{r m s}(y) p_{r m s}\left(y^{\prime}\right)\right]$ in Fig. 5, the wall-normal extent of the structures increases due to roughness at locations away from the wall, and decreases close to the wall. These results illustrate that roughness introduces a wide range of length scales in the pressure-related structures, which are not evident from the velocity-related quantities alone.

This study is offered as a contribution toward the development of systematic and well-defined metrics to quantify the effect of surface roughness on the outer layer. The pressure statistics reveal that both velocity and pressure data are needed to obtain a complete picture of inner/outer interactions for a rough-wall-bounded turbulence.

${ }^{1}$ K. Bhaganagar, J. Kim, and G. Coleman, "Effect of roughness on wallbounded turbulence," Flow, Turbul. Combust. 72, 463 (2004).

${ }^{2}$ A. E. Perry, K. L. Lim, and S. M. Henbest, "An experimental study of the turbulence structure in smooth- and rough-wall boundary layers," J. Fluid Mech. 177, 437 (1987).

${ }^{3}$ M. R. Raupach, R. A. Antonia, and S. Rajagopalan, "Rough-wall turbulent boundary layers," Appl. Mech. Rev. 44, 1 (1991).

${ }^{4}$ J. George and R. L. Simpson, "Some effects of sparsely distributed three- 
dimensional roughness elements on two-dimensional turbulent boundary layers," AIAA Pap. 2000-0915 (2000).

${ }^{5}$ K. S. Choi and N. Fujisawa, "Possibility of drag reduction using d-type roughness,” Appl. Sci. Res. 50, 315 (1991).

${ }^{6}$ F. Faruya, "Turbulent boundary layer and flow resistance on plates roughened by wires," J. Fluids Eng. 98, 635 (1976).

${ }^{7}$ S. Leonardi, P. Orlandi, R. Smalley, L. Djenidi, and R. Antonia, "Direct numerical simulations of turbulent channel flow with transverse square bars on one wall," J. Fluid Mech. 491, 229 (2003).

${ }^{8}$ P. J. Mulhearn, "Turbulent boundary layer wall-pressure fluctuations downstream from an abrupt change in surface roughness," Phys. Fluids 19, 796 (1976).
${ }^{9} \mathrm{~J}$. Kim, "On the structure of pressure fluctuations in simulated turbulent channel flow," J. Fluid Mech. 205, 421 (1989).

${ }^{10}$ J. Kim, P. Moin, and R. D. Moser, "Turbulence statistics in fully developed channel flow at low Reynolds number," J. Fluid Mech. 177, 133 (1987).

${ }^{11}$ W. K. Blake, Turbulent-boundary layer pressure fluctuations over rough walls. Proceedings of the 78th Meeting of the Acoustical Society of America (Acoustical Society of America, New York, 1969), p. 45.

${ }^{12} \mathrm{~A}$. Thomas and M. Bull, "On the role of wall pressure fluctuations in deterministic motions in the turbulent boundary layer," J. Fluid Mech. 128, 283 (1983). 